\title{
Elfe XX-XXI
}

ELFe XX-XXI

Études de la littérature française des XXe et XXle siècles

$10 \mid 2021$

Modes de Présence et Fonctions de l'écrivain dans la cité

\section{Peut-on penser une posture collective ? Tentative de théorisation à travers l'exemple du collectif inculte}

Can we imagine a collective posture? An attempt at theorisation through the example of the Inculte collective

Jean-Marc Baud

\section{OpenEdition}

Journals

Édition électronique

URL : https://journals.openedition.org/elfe/3364

DOI : $10.4000 /$ elfe.3364

ISSN : 2262-3450

Éditeur

Société d'étude de la littérature de langue française du XXe et du XXIe siècles

\section{Référence électronique}

Jean-Marc Baud, « Peut-on penser une posture collective ? Tentative de théorisation à travers

l'exemple du collectif inculte », Elfe XX-XXI [En ligne], 10 | 2021, mis en ligne le 15 octobre 2021 consulté le 22 octobre 2021. URL : http://journals.openedition.org/elfe/3364 ; DOI : https://doi.org/ $10.4000 /$ elfe.3364

Ce document a été généré automatiquement le 22 octobre 2021 


\title{
Peut-on penser une posture collective? Tentative de théorisation à travers l'exemple du collectif inculte
}

\author{
Can we imagine a collective posture? An attempt at theorisation through the \\ example of the Inculte collective
}

Jean-Marc Baud

1 Le recours au concept de posture s'est considérablement développé dans le champ critique depuis une dizaine d'années, en particulier pour ce qui est de l'analyse des modes de présence de l'écrivain contemporain. Cette notion a d'abord été théorisée par Jérôme Meizoz, à la suite de propositions d'Alain Viala, dans un ouvrage paru en 2007 : Postures littéraires. Mises en scène modernes de l'auteur. Dans sa définition minimale, la posture auctoriale est « la manière singulière d'occuper une "position" dans le champ littéraire ${ }^{1} »$. Elle permet ainsi de mener de front analyse interne des œuvres et analyse externe du contexte, comme l'explique Jérôme Meizoz: "En parlant de "posture" d'auteur, on veut décrire relationnellement des effets de texte et des conduites sociales. Autrement dit, sur un plan méthodologique, cette notion articule la rhétorique et la sociologie ${ }^{2} »$. Permettant d'analyser les différents modes de singularisation auctoriale, elle est appliquée prioritairement à des œuvres autobiographiques ou autofictionnelles par son principal théoricien, qui analyse notamment les postures de Romain Gary, JeanJacques Rousseau ou encore Michel Houellebecq.

2 À rebours de cette corrélation entre posture et singularité, nous proposerons quelques éléments de légitimation du concept de posture collective avant d'esquisser les traits d'une telle posture dans le champ contemporain à travers l'exemple du collectif Inculte, qui est sans doute le groupe littéraire le plus important des dernières décennies en France ${ }^{3}$. Il se compose d'une dizaine de romanciers, éditeurs, philosophes, traducteurs ${ }^{4}$ rassemblés à partir de 2004 autour d'une revue, puis de la maison d'édition du même nom. Entre humour burlesque et parodique, excursions vers la littérature 
étrangère et exercices de philosophie buissonnière à la fois joueurs et sérieux, les vingt numéros d'Inculte, tout comme les ouvrages collectifs qui la prolongent ont été un lieu de formation décisif pour les membres du groupe, dont la plupart ont accédé aujourd'hui à la reconnaissance, voire à la consécration (Maylis de Kerangal, Mathias Énard). Nous souhaiterions montrer ici que la notion de posture collective peut s'avérer utile pour l'étude d'un groupe dont l'identité s'est construite en dehors des outils traditionnels d'identification des regroupements artistiques (manifeste, programme ou chef de file) et qui appelle donc de nouveaux modes de saisie pour repérer la façon dont s'y construit du commun.

\section{La posture collective : état des lieux d'un impensé}

Chez Jérôme Meizoz, la posture semble indissociable de l'idée de singularité. Le mot apparaît dans la définition minimale qu'il donne de ce concept, et constitue même le titre de son deuxième ouvrage sur la question: La Fabrique des singularités: postures littéraires II. Pour le chercheur, l'articulation entre position et style énonciatif « est toujours singulière ${ }^{5}$ ", et elle l'est d'autant plus en littérature contemporaine où la multiplication des prestations publiques amène les écrivains à se présenter comme de " grands singuliers ${ }^{6}$ ». Posture collective sonnerait donc comme un oxymore, la notion paraissant, de fait, inemployable pour l'étude des groupes littéraires. Jérôme Meizoz la distingue pour cette raison de celle de «scénographie ", telle qu'elle est proposée par José-Luis Diaz dans L'Écrivain imaginaire. Scénographies auctoriales à l'époque romantique ${ }^{7}$, mieux à même, selon lui, de permettre l'analyse des figures et des positionnements collectifs en littérature :

Mais on voit aussi que les notions de scénographie et de posture visent des niveaux différents : la scénographie est un fait générique et collectif, propre, par exemple, à telle école ou mouvement. La posture, quant à elle, désigne la singularisation d'un positionnement auctorial : une tentative de se présenter comme unique, hors de toute appartenance ${ }^{8}$.

\section{Approches collectives de la posture}

4 Pourtant, cette notion n'exclut pas toute dimension collective. Tout d'abord en tant qu'elle met en jeu une multiplicité d'acteurs et d'instances qui la co-construisent, qu'il s'agisse des éditeurs, des médias, des pairs ou des critiques. D'autre part, si chaque écrivain tend à singulariser sa posture, il puise en même temps dans ce que Jérôme Meizoz nomme un "répertoire historique ${ }^{9}$ » la figure de l'écrivain-citoyen engagé ou du génie malheureux constituent autant de " familles posturales ${ }^{10}$ » auxquels l'écrivain s'affilie, consciemment ou inconsciemment. Peut-on passer, toutefois, de cette dimension générique et verticale (historique) de la posture à l'horizontalité d'une posture groupale?

5 Ici et là, des tentatives d'expérimentation et de théorisation embryonnaires apparaissent depuis quelques années en ce sens, comme le montrent les titres de certains articles ou communications: "Posture collective et survie organisationnelle. L'exemple de la fondation de L'Académie canadienne-française ${ }^{11} »$ d'olivier Lapointe, «Mai 68, les avatars d'une posture générationnelle: ou comment Dany le Rouge est passé aux Verts ${ }^{12}$ ", de Sarah Sindaco, ou encore "Des postures collectives? Sur les groupes littéraires fin de siècle ${ }^{13}$ ", prononcé par Denis Saint-Amand en 2013 à 
Lausanne. En 2009, le numéro 6 de la revue COnTEXTES intitulé «Qui a lu boira. Les alcools et le monde littéraire ${ }^{14} »$ fait une large place à l'hypothèse d'une posture collective. On y compte en effet une dizaine d'occurrences de l'expression, appliquées aux postures surréaliste, situationniste ou bohème. Souvent mentionnée, elle y est cependant peu théorisée. On notera la proposition de définition d'Anthony Glinoer qui, s'arrêtant sur l'« orgie bohème ", analyse la posture collective comme "un réseau de figurations enté sur la position d'un groupe social polymorphe ${ }^{15}$ ». Michel Lacroix propose quant à lui « d'introduire la série [...] des "postures de partage" ou "posture partagées ${ }^{16 "}$ " en s'intéressant à la configuration éthylique des situationnistes. Il invite ainsi à « disposer d'un inventaire des diverses postures "du collectif" propres à la vie littéraire, mais aussi d'études se penchant sur les confrontations/négociations entre individus et groupes, telles que mises en discours et en texte », cette « dialectique entre singularité et collectivité ${ }^{17}$ » constituant l'intérêt majeur de ce concept pour lequel il ouvre quelques pistes de réflexion. On trouve encore d'autres jalons théoriques chez Jérôme Meizoz lui-même, dans un entretien réalisé avec David Martens et publié dans La littérature " en personne » : scènes médiatiques et formes d'incarnation:

Il y a donc, par agrégation ou couches, des répertoires posturaux disponibles et les auteurs les intériorisent au cours de leur socialisation à la vie littéraire. Il me semble que ces répertoires peuvent être collectivement convoqués lorsqu'un ensemble d'auteurs se donne comme groupe, par l'énonciation manifestaire notamment. La publication d'un manifeste et la forte mise en scène des activités collectives (pensons au déroulement très codifié d'une soirée futuriste puis dada) contribuent à poser une norme d'action et d'énonciation, même si elle n'est pas entièrement explicitée. Le plus frappant, ce sont les conduites de vie surréalistes étudiées avec soin par David Vrydaghs. Mais on peut dire de même pour les cénacles romantiques (Anthony Glinoer, Pascal Brissette), le groupe zutiste ou plus tard les futuristes (Henri Béhar) et situationnistes. Le groupe propose une posture: un ethos énonciatif prophétique, péremptoire et violent, chez les surréalistes, accompagné de conduites vestimentaires, gestuelles, d'un rapport à l'alcool par exemple. Mais chaque auteur se réapproprie singulièrement ces injonctions (c'est pourquoi je parle de fabrique des singularités) et joue en quelque sorte sa propre partition. D'où, à mon sens, les fameuses «excommunications » surréalistes qui réagissent à une mauvaise interprétation de la norme posturale implicite ${ }^{18}$.

\section{Quelques pistes théoriques pour la définition d'une posture collective}

6 À la suite de ces réflexions, nous aimerions contribuer à la légitimation du concept de posture collective et formuler quelques traits définitoires de la notion. Une première justification est d'ordre sociologique : la posture est indissociablement liée à la notion de position. Or, tout groupe littéraire occupe une position dans le champ, en concurrence avec d'autres agents collectifs ou individuels. Il est donc nécessaire de faire l'hypothèse qu'il produit dans le même temps une posture collective, qui serait la manière singulière de jouer de cette position (commune). D'autre part, le groupe littéraire est le lieu par excellence de jonction entre des conduites et des discours, entre des pratiques, des sociabilités (soirées, performances, actes de violence, interventions publiques et politiques) et des productions collectives (tracts, manifestes, fictions, revues...), jusqu'à proposer, dans le cas des avant-gardes, un programme «art-vieaction ${ }^{19}$ ", selon l'expression du fondateur du futurisme, Marinetti. L'étude des groupes littéraires se fait nécessairement à la confluence de la dimension rhétorique et 
actionnelle et appelle donc le concept de posture. Et ce d'autant plus qu'il emprunte beaucoup à la métaphore théâtrale: Jérôme Meizoz la décrit tour à tour comme « masque $^{20}$ », « performance ${ }^{21}$ » ou « dispositif scénique ${ }^{22}$ ». De la bataille d'Hernani aux soirées dadaïstes, les groupes littéraires ont beaucoup joué de cette théatralité, contribuant même à inventer les genres de la performance ou du happening. En art, le groupe se donne ainsi comme mise en scène, comme en témoigne la mythification des photos de groupe d'écrivains. La mobilisation du concept de posture collective s'avère d'autant plus intéressante pour l'étude des groupes contemporains que, comme l'a noté Jérôme Meizoz, les lieux et les formes d'intervention de l'écrivain se sont démultipliés (interviews, réseaux sociaux, festivals, résidences, rencontres et signatures, lectures publiques...) dans un champ marqué par une «injonction à la visibilitée ${ }^{3} »$ de plus en plus forte qui tend à faire de l'écrivain « une marque ${ }^{24} »$.

7 L'enjeu d'un tel outil théorique ne serait pas d'étouffer toute posture individuelle mais au contraire d'articuler de façon dynamique son étude à celle des postures collectives, qui ont, comme l'a bien souligné Michel Lacroix, «l'intérêt particulier de montrer les écrivains aux prises avec la double contrainte de la singularité et de la collectivité : comment se distinguer, comme écrivain, tout en adhérant à un groupe ${ }^{25}$ ?». On jouerait ainsi d'une posture collective comme un groupe de rock joue un concert, chacun suivant sa "partition » posturale dans un ensemble composé d'unissons, d'harmonies, de solos et de couacs. Elle résulterait ainsi du frottement de forces centrifuges (la tendance singularisante de la posture auctoriale) et de forces centripètes, que furent exemplairement dans l'histoire littéraire le manifeste, la figure du leader ou l'identification d'un adversaire. Ces moteurs de cohésion ou d'alignement postural peuvent ainsi, grâce à cet outil, être étudiés de façon cohérente et complète, depuis le mimétisme vestimentaire des membres de certains cénacles vis-à-vis de leur maitre. Il devient aussi possible d'analyser en quoi la virulence de l'ethos et du ton manifestaires peut être liée à l'éloge, voire à la pratique de la violence. Mais la posture collective permet aussi de ne pas limiter l'identification des normes communes à celles fixées par les seules autorités traditionnelles (texte-cadre ou chef de file), qui établiraient un dogme esthétique ou politique indiscutable. D'autres marqueurs posturaux pourraient être analysés, comme l'affiliation à un répertoire groupal préexistant (posture ludique, éthylique, révolutionnaire...), l'adoption d'ethos préférentiels reliant les œuvres des différents écrivains du groupe, ou la constitution d'une bibliothèque commune, faisant le lien entre postures collective et individuelles et ethos énonciatifs des différents membres du groupe, revendiquant et exploitant singulièrement les références ainsi élues.

8 Dès lors, comment articuler postures collective et individuelle ? Quel écart à la norme est-il toléré dans les groupes? Quelle part de réappropriation individuelle est-elle envisageable? David Martens proposait, dans l'entretien avec Jérôme Meizoz, de nommer ces écarts possibles des « latitudes posturales ${ }^{26}$ » et de réfléchir à une possible typologie des groupes selon leur degré de tolérance à ces latitudes : école, mouvement, collectif... S'instaure ainsi un subtil jeu stratégique pour les écrivains entre un alignement permettant de bénéficier de la visibilité offerte par le groupe et un nécessaire jeu de distinction. On pourrait faire l'hypothèse que, loin de recouvrir les postures individuelles, la posture collective tend au contraire, à des rythmes différents, à approfondir, renforcer, creuser leur singularisation par la fréquentation intense des autres membres et l'hypervigilance vis-à-vis de leurs positionnements intellectuels, 
politiques et esthétiques qui tendent à faire du groupe un champ dans le champ. En ce sens, la notion pourrait être utile pour étudier les cas de rupture analysés comme autant de discordances posturales qui articuleraient esthétique et sociologie, sans les réduire à la première explication, souvent secondaire mais survalorisée dans les études littéraires, ni à la seconde, qui attribue ces départs de façon parfois trop mécanique à une différence de capital social ou d'habitus.

9 La posture collective constitue donc, selon nous, un outil global, cohérent et prometteur de saisie des phénomènes collectifs en littérature, dont nous nous proposons ici de tester la portée théorique.

\section{Posture groupale en littérature contemporaine : l'exemple du collectif Inculte}

10 À partir de ces premiers éléments de réflexion, nous aimerions essayer de cerner les modes d'énonciation et de production d'une posture collective et les lieux où elle se construit. Nous prendrons pour cela l'exemple du collectif Inculte.

\section{Leitmotive incultes}

11 Une première difficulté réside dans l'absence de ces moteurs de cohésion posturale dont nous parlions : les incultes se plaisent en effet à répéter qu'ils se refusent à écrire un manifeste et à faire émerger une figure de leader :

Arno Bertina : «Dès le premier numéro, on nous a suggéré d'écrire un manifeste. [...] Nous nous sommes alors demandé pourquoi nous n'y avions pas pensé. La réponse est simple : [...] parce qu'il y a quelque chose de détestable à se dire que, l'échiquier ayant soixante-quatre cases, nous allions en inventer ou bien occuper la trente-deuxième ou la première. "Nous allons nous opposer à la génération de la revue Perpendiculaire ", par exemple. C'est idiot, et ça ne rend pas compte des vitalités possibles à l'intérieur du champ littéraire. Ensemble, nous avons compris qu'il n'y avait aucune génération contre laquelle nous souhaiterions nous élever. [...] Donc, pas de manifeste, pas de petite cuisine idiote. Les grandes phrases et les grands programmes esthétiques viennent aux littéraires avec une facilité très agaçante, on fait des phrases qu'on dresse comme des barricades. Ce travers est ridicule, peu intéressant ${ }^{27}$ »

Maylis de Kerangal : «Liberté par rapport à l'idée de manifeste qu'il n'était pas question d'écrire. Liberté par rapport à la question des avant-gardes, liberté par rapport à la question d'une école. Nous ne serions pas une école, nous serions des écrivains, chacun ayant à la fois une trajectoire autonome, un travail autonome, et en même temps nous nous retrouverions ensemble pour travailler ${ }^{28}$ ”

Oliver Rohe : «La seule ligne était de ne pas en avoir, de ne pas débarquer dans le champ littéraire avec un manifeste, une volonté d'avant-garde, autant de choses auxquelles on ne croit plus et qui sont inefficaces ${ }^{29}$ "

La répétition et la convergence de ces refus (du manifeste, de la guerre des générations, de l'école) en font alors des marqueurs posturaux, permettant à Inculte de se distinguer du répertoire avant-gardiste et de présenter le groupe comme un espace de liberté à la fois esthétique et politique. À défaut de ces centres d'énonciation de la posture collective que sont le manifeste ou la figure du leader, sa production passera justement par de tels leitmotive qui déploient un réseau conceptuel et lexical commun donnant au collectif sa cohérence. La formule, amplement répétée par ses membres, d'un 
collectif « à géométrie variable ${ }^{30}$ » ou la revendication d'une littérature " potache ${ }^{31}$ ", qui imprègne leurs déclarations tout comme le discours journalistique et critique tenu sur Inculte, constituent d'autres exemples de ces leitmotive.

\section{Les multiples visages de l'enquêteur inculte}

La posture collective se construit aussi à travers l'adoption d'ethos préférentiels par les auteurs du groupe. Nous nous intéresserons ici en particulier à la figure de l'enquêteur qui nourrit la pratique et l'écriture des différents membres d'Inculte. Son émergence et sa circulation se font par deux biais principaux. Elles passent d'abord par la constitution d'une bibliothèque en partage, pouvant induire des dynamiques de convergences esthétiques. L'intérêt collectif porté à W. G. Sebald par les incultes a pu encourager la pratique de la littérature documentaire chez les romanciers. Le dossier du premier numéro de la revue Inculte porte en effet sur l'écrivain allemand qui fait sept ans plus tard l'objet d'une monographie publiée chez Inculte, à laquelle plusieurs membres du collectif participent, témoignant de l'influence importante qu'a jouée l'écrivain sur leurs œuvres. L'émergence de cet ethos privilégié passe également par les productions collectives d'Inculte, et en particulier sur cette question, l'écriture du deuxième volume des Devenirs du roman. Publié en 2014 et sous-titré "Écriture et matériaux", l'ouvrage réunit les contributions de vingt-quatre romanciers réfléchissant à la place et à l'usage du document dans leur écriture. En publiant ce livre à l'occasion des dix ans de la maison d'édition et en choisissant d'y contribuer individuellement, les incultes lient leurs trajectoires singulières, collective et éditoriale à la question documentaire. Dans cet ouvrage se dessine en filigrane, au fil de leurs contributions, une galerie voire un bestiaire inculte de la pratique documentaire.

L'ethos de l'enquêteur est ainsi décliné selon le projet des différents romanciers qui sélectionnent les pratiques et la panoplie auctoriales qui leur conviennent. Dans Une île, une forteresse $e^{32}$, publié en 2016 chez Inculte, Hélène Gaudy mène l'enquête sur la ville de Terezín, ancien ghetto juif durant la Seconde guerre mondiale. Elle marche sur les traces de l'auteur d'Austerlitz, W. G. Sebald, qu'elle a découvert grâce à Inculte, à l'occasion de sa participation à la monographie ${ }^{33}$ que lui consacre la maison d'édition en 2011. Le collectif a ainsi joué comme "déclencheur objectif du livre ${ }^{34}$ ", selon les mots de l'autrice qui s'invente, dans la filiation sebaldienne, une figure de narratrice arpenteuse et cartographe. Le lieu est en effet «le point précis où commence un roman ${ }^{35}$ ", écrit-elle dans " Sur les lieux : construire, fabriquer, se repérer, arpenter ", contribution au Devenirs du roman dans laquelle elle présente son protocole d'enquête, deux ans avant la publication de l'ouvrage. Les quatre verbes qui composent son titre auxquels on pourrait ajouter "collecter" - sont autant de gestes mis en place par l'enquêtrice pour écrire ce livre où se mêlent déambulations, descriptions de photographies et de films de propagande nazie ou encore témoignages des habitants de la ville. Le dispositif labyrinthique rejoue les dérives de la narratrice et construit Terezín comme un palimpseste de temporalités et de formes qui seul, peut-être, permet de dire la ville, à l'opposé de la clarté trompeuse de la propagande nazie qui en avait fait un ghetto «modèle». Dans les Devenirs du roman, Mathieu Larnaudie campe une autre figure d'enquêteur à côté de celle d'Hélène Gaudy: le "sémionaute ${ }^{36}$ ». Cette figure rend compte des nouvelles expériences documentaires de l'écrivain à l'heure du numérique et du big data. Les Effondrés ${ }^{37}$ décrit ainsi cette navigation parmi tous les signes qui constituent le récit médiatique de la crise financière de 2008, entre vidéos en 
ligne, articles de presse et chaînes d'information en continu. Dans ce livre scandé par l'expression "on vit ", qui revient plus d'une dizaine de fois, narrateur et lecteur se font téléspectateurs suivant le zapping d'une crise en direct où défilent Nicolas Sarkozy, Bernard Madoff, Alan Greenspan, Angela Merkel... La tâche du sémionaute est alors d'agencer ces éléments pour rendre intelligible ce grand flux d'informations et de capitaux que constitue la dépression financière. Surtout, il s'attache à contrer la narration dominante par une syntaxe alternative, empruntant volontiers à Claude Simon et qui, par son ampleur, sa complexité et sa sensorialité démonte l'édifice idéologique néolibéral. Cette phrase vient ainsi démythifier le récit de la crise en prenant comme contre-modèles tout à la fois la concision expéditive des bandeaux défilants des chaînes d'information en continu et la catharsis hollywoodienne préservant «l'hégémonie idéologique » grâce au sacrifice d'un bouc-émissaire, "méchant de cinéma, [...] escroc superlatif ${ }^{38}$ ". La posture d'enquêteur d'Arno Bertina est elle aussi politique. Là où Mathieu Larnaudie s'attache à décoder les mythologies des puissants, dans une approche sémiologique, Arno Bertina ancre sa posture dans des pratiques d'enquêtes menées au plus près des plus démunis et des invisibles, qu'il s'agisse de jeunes prostituées congolaises dans L'Âge de la première passe (2020), d'un sans-domicile fixe dans La Borne SOS 77 (2009) ou d'ouvriers licenciés dans Des châteaux qui brûlent (2017). Si ces livres n'appartiennent pas tous au genre de l'enquête, ils reposent sur des expériences de terrain et un important travail de recueil de voix qui inscrit l'auteur dans les traces de François Bon ou de Svetlana Alexievitch, à qui Arno Bertina a consacré l'édition 2017 des Rencontres de Chaminadour dont il était le conseiller littéraire. L'Âge de la première passe mêle ainsi la voix de l'auteur et celles des jeunes prostituées auprès desquelles il a mené des ateliers d'écriture à partir de 2014 au Congo, à l'invitation de l'ONG Actions de solidarité internationale. Ni représentant, ni porte-parole, l'enquêteur ne cesse de s'interroger sur sa propre position et les possibles émancipateurs de son geste, en évitant tout surplomb dans un livre qui s'écrit dans la doublure des mots de ces jeunes filles. Arno Bertina dessine ainsi une posture d'enquêteur-citoyen engagé qui se manifeste non seulement dans la genèse et la forme de ses livres, mais aussi dans différents actes militants qui les prolongent. Dans la foulée de la publication des Châteaux qui brûlent, qui raconte la séquestration d'un secrétaire d'État par les salariés d'une usine prête à fermer, l'écrivain a par exemple rencontré à plusieurs reprises des ouvriers licenciés de GM\&S pour leur apporter son soutien et recueillir leur témoignage ${ }^{39}$.

15 Cartographe, sémionaute ou enquêteur-militant, les incultes tissent ainsi leur posture propre dans l'écheveau collectif.

\section{Autoportrait des incultes en buveurs}

16 Nous souhaiterions enfin donner un aperçu des différents lieux possibles de construction d'une posture partagée dans le champ contemporain, en nous intéressant au développement d'une posture festive chez Inculte. L'éthylisme apparaît en effet comme l'un des traits centraux du collectif que ses auteurs ne manquent pas d'exposer dans les différents lieux où leur camaraderie se manifeste, de la revue à Facebook, des remerciements aux festivals. 2011. Le choix de consacrer le dernier numéro de la revue à ce thème est un indice de 
son importance posturale, de sa place dans la sociabilité du groupe et une façon d'entériner définitivement une éthique qui est aussi une esthétique, la pratique de la littérature comme profanation et comme joie. À l'intérieur de ce dossier, l'article de Maylis de Kerangal, "Notre sélection », se présente comme une liste de recettes de cocktails à mi-chemin du document et de la fiction. Le possessif indique bien que le texte compose une forme d'autoportrait des incultes en buveurs invétérés, achevant la revue sur une représentation des incultes non pas dans la bibliothèque ou à l'étude, mais au bar. L'un des cocktails décrits, "La femme indécise ", fait l'objet d'une contribution de Mathieu Larnaudie qui en conte la genèse :

Je ne sais presque rien sur ce baptême, si ce n'est qu'il eut lieu dans un bar bruxellois, et qu'on le doit à Claro et Mathias Énard. C'est ce dernier qui m'en délivrait le faire-part, une nuit où nous nous étions retrouvés dans un haut lieu de nos habitudes qui ne ferme qu'à 8 heures du matin. Tandis que quelques vodkaschampagne transitaient par des mains tendues, depuis celles du serveur jusqu'à leurs destinataires: "On a décidé d'appeler ça une femme indécise. La femme indécise est née à l'Archiduc, rue Antoine Dansaert, d'un père ivrogne et d'une mère prude. » Sur l'instant, je trouvais cela tout à fait normal (c'était l'un de ces instants où l'on trouverait tout normal), et me promettais d'adopter dorénavant cette désignation pour notre breuvage emblématique, bien conscient tout de même, évidemment, qu'il faudrait à chaque fois expliquer au barman à qui on la commanderait de quoi celle-ci se composait ${ }^{40}$.

Par ses tournures et son propos, "Note sur la naissance de la femme indécise » s'apparente ainsi à un geste, en partie ironique, de mythologisation proche des récits cénaculaires du XIX ${ }^{e}$ siècle. L'identification d'un "breuvage emblématique " prolonge cet acte de symbolisation par la bouteille, qui court dans tout le dossier. L'exposition d'une camaraderie éthylique circule aussi dans les marges des récits de ses auteurs, chez Arno Bertina notamment, qui remercie, à la fin de Je suis une aventure, les "incultes qui m'ont fait boire ${ }^{41}$ ", ou évoque dans la postface qu'il donne à Pôle de résidence momentanée, les "nuits à faire le $\operatorname{con}^{42}$ " avec Mathieu Larnaudie et le lendemain "qui a toujours une gueule de bois ${ }^{43}$ ». L'éthylisme est également présenté dans les articles qui portent sur Inculte comme un trait essentiel de leur sociabilité, depuis le "souvenir de soirée alcoolisé ${ }^{44}$ " évoqué dans Les Inrocks, à la mention de la présence de "bières, parce que c'est samedi soir ${ }^{45}$ " dans L'Obs, jusqu'aux propos d'oliver Rohe qui fait de la revue le « prétexte » d'une « massive absorption d'alcool ${ }^{46}$ ». Cités dans l'article "Des incultes bien en plumes ", les écrivains réaniment même en partie l'imaginaire dionysiaque de l'ivresse inspirante: "On est tous partants pour penser qu'une bonne idée arrive souvent tard dans la soirée et qu'il n'en reste souvent que deux à s'en souvenir ${ }^{47}$ ». Les sociabilités littéraires auxquelles ils participent sont aussi scandées par ces rituels, depuis une soirée du festival de Bron en 2004 qui fonde l'amitié entre François Bégaudeau, Oliver Rohe et Arno Bertina, jusqu'à la consécration de Mathias Énard, fêtant son prix Goncourt en 2015 avec ses camarades d'Inculte, comme le raconte Baptiste Liger dans L'Express:

Le «nouveau Balzac» [...] rejoint alors ses copains d'Inculte dans le jardin - où il pleut un peu. Un journaliste les observe et, philosophe, résume l'avis de nombre de convives: «S'ils partent ensemble pour la tournée des grands ducs, ça n'est pas gagné pour Léa Salamé demain matin ${ }^{48} . . . »$

19 Enfin, cette scénographie festive se déploie aussi sur Internet, en particulier sur les réseaux sociaux à travers le jeu des commentaires, des posts et des dédicaces. En réaction au post d'anniversaire publié sur Facebook par la maison d'édition Inculte pour ses douze ans d'existence, Arno Bertina écrivait ainsi un commentaire tout rabelaisien : 
«Il faudrait boire beaucoup pour fêter ça. Mais où trouver assez? À Rungis carrément ? ", auquel Jérôme Schmidt répondait : "À la Mère Agitée bien sûr ${ }^{49}$ ! ». Il en va de même d'un post de Mathias Énard, publié le 12 octobre 2017, qui reproduisait la photographie d'une notice sur son livre L'Alcool et la nostalgie traduit en allemand. Ce post était l'occasion d'une double dédicace, la notice étant signée de Mathias Énard avec la mention « Pour Inculte : amitiés les $\mathrm{amis}^{50}$ » et sa publication sur Facebook étant de plus accompagnée de tags mentionnant les membres du collectif. Dédicacée à la maison d'édition qui édita l'ouvrage en 2011 mais aussi au collectif et, de façon implicite, aux soirées communes passées ensemble, cette publication illustre bien la façon dont la camaraderie numérique rejoue la scène d'ivresse sur la toile.

La posture éthylique du collectif Inculte s'inscrit dans la longue histoire des groupes littéraires, comme l'a bien montré le numéro 6 de la revue ConTextes intitulé « Qui a lu boira » où sont décrites les conduites de vie éthyliques surréaliste ${ }^{51}$, situationniste $^{52}$ et bohème ${ }^{53}$. Les deux directeurs du numéro rappellent, en introduction, que «la consommation d'alcool y est abordée comme une pratique qui cimente la scénographie collective des groupes littéraires tout en influençant leur esthétique ${ }^{54} »$, une phrase qui pourrait tout aussi bien s'appliquer au collectif Inculte qui, alors qu'il tend plutôt à s'en distinguer dans sa posture, se réaffilie par là à l'histoire des groupes littéraires et des avant-gardes.

\section{Conclusion}

21 S'il est «sage de ne pas l'employer à tout va ${ }^{55}$ ", comme le recommande Alain Viala, le concept de posture semble prometteur pour l'étude des groupes littéraires, permettant une saisie transversale et précise des phénomènes collectifs, à condition de qualifier ces gestes posturaux, de les inscrire dans l'histoire littéraire et d'articuler finement discours et conduites, texte et hors-texte. La posture festive d'Inculte traverse ainsi ethos narratoriaux et discours des auteurs, se manifeste dans les livres individuels et collectifs, dans la revue ou le paratexte mais encore sur Internet ou lors de leurs interventions publiques. Les leitmotive incultes (groupe à géométrie variable, tonalité potache, refus du manifeste...) composent également un réseau postural dessinant un ensemble de convergences hors de tout texte-cadre. À la fois cohérente et plurielle, la posture permet d'articuler l'individuel et le collectif pour l'étude des groupes littéraires. Elle résulte du jeu entre ces deux niveaux, chacun des membres interprétant, au sens musical du terme, les traits posturaux communs. Des ethos préférentiels, celui de l'enquêteur dans le cas des incultes, s'élaborent ainsi au contact d'une bibliothèque en partage et d'actes collectifs d'écriture, et sont réappropriés singulièrement par les membres du groupe dans leurs phrases, leurs livres et leurs prises de position.

22 Pour finir, si la notion de posture collective semble tout à fait adaptée à l'étude d'Inculte, nous pensons qu'elle pourrait être appliquée utilement aux autres collectifs contemporains et aux groupes des siècles précédents, offrant l'occasion d'une relecture renouvelée et globale de ces phénomènes décisifs de l'histoire littéraire. 


\section{NOTES}

1. Jérôme Meizoz, Postures littéraires. Mises en scène modernes de l'auteur, Genève, Slatkine, 2007, p. 18.

2. Ibid., p. 21.

3. En témoigne le récent colloque qui lui a été consacré à la Sorbonne Nouvelle les 7 et 8 février 2020 : «Inculte. Pratiques éditoriales, gestes collectifs et inflexions esthétiques », organisé par Aurélie Adler, Jean-Marc Baud, Alexandre Gefen et Laurent Demanze.

4. Ce collectif à "géométrie variable", comme le présente son site internet, a regroupé aujourd'hui, autour d'une revue et d'ouvrages collectifs adossés à la maison d'édition du même nom, Bruce Bégout, Arno Bertina, Claro, Alexandre Civico, Mathias Énard, Hélène Gaudy, Maylis de Kerangal, Mathieu Larnaudie, Stéphane Legrand, Nicolas Richard, Oliver Rohé, Jérôme Schmidt et, anciennement, Joy Sorman et François Bégaudeau.

5. Jérôme Meizoz, «La fabrique de la posture (Dialogue avec David Martens) », in Jérôme Meizoz, La littérature "en personne »: scènes médiatiques et formes d'incarnation, Genève, Slatkine, 2016, p. 173.

6. Ibid., p. 27.

7. Voir José-Luis Diaz, L'Écrivain imaginaire. Scénographies auctoriales à l'époque romantique, Paris, Honoré Champion, 2007.

8. Jérôme Meizoz, La Littérature "en personne» : scènes médiatiques et formes d'incarnation, op. cit., p. 12.

9. Ibid., p. 83.

10. Jérôme Meizoz, Postures littéraires. Mises en scène modernes de l'auteur, op. cit., p. 25.

11. Olivier Lapointe, « Posture collective et survie organisationnelle. L'exemple de la fondation de L'Académie canadienne-française ", in Denis Saint-Amand (dir.), La Dynamique des groupes littéraires, Liège, Presses Universitaires de Liège, 2016, p. 143-153.

12. Sarah Sindaco, «Mai 68, les avatars d'une posture générationnelle », COnTEXTES, $n^{\circ} 8,2011$. En ligne, URL : https://journals.openedition.org/contextes/4718 [consulté le 1 novembre 2019].

13. Denis Saint-Amand, "Des postures collectives? Sur les groupes littéraires fin de siècle", communication prononcée lors du colloque Figures, ethos et posture de l'auteur au fil des siècles, organisé à l'Université de Lausanne, les 20 et 21 juin 2013.

14. Geneviève Boucher et Pascal Brissette (dir.), "Qui a lu boira. Les alcools et le monde littéraire », COnTEXTES, $\mathrm{n}^{\circ}$ 6, septembre 2009. En ligne, URL : http://journals.openedition.org/ contextes/4458 [consulté le 19 janvier 2020].

15. Anthony Glinoer, «L'orgie bohème », COnTEXTES, $n^{\circ} 6$, septembre 2009, op. cit.

16. Michel Lacroix, «Un sujet profondément imprégné d'alcool », COnTEXTES, $\mathrm{n}^{\circ} 6$, septembre 2009. En ligne, URL : https://journals.openedition.org/contextes/4433 [consulté le 8 juillet 2021].

17. Ibid.

18. Jérôme Meizoz, «La fabrique de la posture (Dialogue avec David Martens)», Art. cit., p. 175-176.

19. Cité par Anne Tomiche, La Naissance des avant-gardes occidentales (1909-1922), Paris, Armand Colin, « U », 2015, p. 35.

20. En référence à la notion latine de persona. Voir : Jérôme Meizoz, Postures littéraires. Mises en scène modernes de l'auteur, op. cit., p. 19.

21. Jérôme Meizoz, La Fabrique des singularités. Postures littéraires II, op. cit., p. 9.

22. Jérôme Meizoz, La Littérature "en personne ": scènes médiatiques et formes d'incarnation, op. cit., p. 39.

23. Ibid., p. 22. 
24. Ibid., p. 75.

25. Michel Lacroix, «Un sujet profondément imprégné d'alcool », Art. cit.

26. Jérôme Meizoz, « La fabrique de la posture (Dialogue avec David Martens) », Art. cit., p. 176.

27. Laurent Demanze, «Faim de littérature (Arno Bertina, Pierre Senges, Tanguy Viel). Entretien », in Laurent Demanze, Dominique Viart (dir.), Fins de la littérature : esthétique et discours de la fin, t. 1, Paris, Armand Colin, Recherches, 2012, p. 269.

28. Jean Kaempfer, « Le goût du romanesque, le sens de l'épopée. Maylis de Kerangal s'entretient avec Jean Kaempfer ", in Maylis de Kerangal (dir.), Maylis de Kerangal sur les grands chemins de Claude Simon, Guéret, Carnets de Chaminadour, $\mathrm{n}^{\circ}$ 11, 2016, p. 70.

29. Elisabeth Philippe, « Bande à part », Les Inrocks, n 958, du 9 au 15 avril 2014, p. 47.

30. L'expression est présente sur la page de présentation du collectif Inculte sur le site Internet de l'éditeur. Voir : https://inculte.fr/auteurs/collectif-inculte/ [consulté le 17 janvier 2018]. Elle est également très souvent employée par les membres du collectif, comme par exemple Arno Bertina et Mathieu Larnaudie lors de leur entretien avec Aurélie Adler lors de la journée d'étude "Auteurs en scène. Lieux et régimes de visibilité des écrivains contemporains », organisée le 3 juin 2016 à l'université Paris-Diderot (voir: https://www.fabula.org/colloques/ document4613.php [consulté le 1 août 2019]), ou encore Alexandre Civico lors de la soirée «Écrire en collectif » organisée le 2 mars 2016 au Triangle (Rennes).

31. Le terme revient à de très nombreuses reprises dans le métadiscours des écrivains du collectif, comme nous avons pu le constater lors des entretiens que nous avons menés avec François Bégaudeau (28 août 2019), Mathieu Larnaudie (17 avril 2017) et Arno Bertina (12 avril 2018). Il devient également omniprésent dans sa réception médiatique, comme le montrent ces quelques exemples : «De là une allure d'auberge espagnole, où voisinent humour potache et grand sérieux» (Laurence Marie, Pierre Savy (coord.), « Revues mode d'emploi », Labyrinthe, $\mathrm{n}^{\circ} 31,2008$, p. 66) ; «Rapide, aigu, volontiers piquant ou provocateur, le livre joue de tous les registres, du plus sérieux à la blague de potaches " (Michel Abescat, «Fragments de France François Bégaudeau, Arno Bertina et Oliver Rohe. Une année en France ", Télérama, 28 avril 2007, p. 67) ; «Le mélange des voix et des genres, de l'approche universitaire et du ton potache, de la pensée et de la fiction, [...] voilà ce qui réunit ces trentenaires » (Grégoire Leménager, «Une sacrée bande d'incultes ", L'Obs, 17 avril 2009. En ligne, URL: https://bibliobs.nouvelobs.com/ actualites/20090417.BIB3274/une-sacree-bande-d-incultes.html [consulté le 6 juillet 2016]).

32. Hélène Gaudy, Une île, une forteresse, Paris, Inculte, 2016.

33. Collectif, Face à Sebald, Paris, Inculte, 2011.

34. Entretien personnel réalisé avec l'autrice à Paris le 21 février 2018.

35. Hélène Gaudy, "Sur les lieux : construire, fabriquer, se repérer, arpenter ", in Collectif, Devenirs $d u$

roman. Écriture et matériaux, Paris, Inculte, 2014, p. 193.

36. Mathieu Larnaudie, "Empoigner le monde - Captures et captations - ", Devenirs du roman. Écriture et matériaux, op. cit., p. 88.

37. Mathieu Larnaudie, Les Effondrés [2010], Arles, Actes Sud, « Babel », 2013.

38. Ibid., p. 153.

39. Voir par exemple Arno Bertina, "Qui est responsable?", AOC, 26 mai 2019. En ligne, URL : https://aoc.media/fiction/2019/05/26/qui-est-responsable/ [consulté le 10 mai 2021].

40. Mathieu Larnaudie, "Note sur la naissance de la femme indécise », Inculte, $n^{\circ} 20$, janvier 2011, p. 63-64.

41. Arno Bertina, Je suis une aventure, Paris, Verticales, 2012, p. 492.

42. iId., " Habiter un espace incertain (l'amitié) », in Mathieu Larnaudie

Larnaudie

, Pôle de résidence momentanée, Paris, Les petits matins, 2007, p. 142. 
43. Ibid., p. 139.

44. Elisabeth Philippe, « Bande à part », Art. cit., p. 46.

45. Grégoire Leménager, « Une sacrée bande d'incultes », Art. cit.

46. Caroline Hoctan et Jean-Noël Orengo, « Entretien avec Oliver Rohe », D-Fiction. En ligne, URL : 1 janvier 2010, http://d-fiction.fr/2010/01/entretien-avec-oliver-rohe/ [consulté le 10 mars 2018].

47. Karine Papillaud, «Des "incultes" bien en plumes», Art. cit., 20 minutes, 29 mars 2012, https://www.20minutes.fr/culture/906975-20120329-incultes-bien-plumes [consulté le 16 juin 2017].

48. Baptise Liger, « Mathias Énard et le Goncourt: "J'avais l'impression d'être dans un film de zombies!" ", L'Express, 4 novembre 2015, https://www.lexpress.fr/culture/livre/mathias-Énardet-le-goncourt-j-avais-l-impression-d-etre-dans-un-film-de-zombies_1732741.html [consulté le 10 mai 2016].

49. Page Facebook des éditions Inculte, 17 janvier 2017, https://www.facebook.com/ editions.inculte/?

ref =search\&_tn__ $=\% 2 \mathrm{Cd} \% 2 \mathrm{CPR} \&$ eid =ARBJ0psf6KXsIBIr2ZfbrxhzyzDUBmEt0NWY3jBiRJo1ftIfjDV1RZaV4bv2gLstKQTBbcQHwgQV_q$\mathrm{v}$ [consulté le 18 janvier 2017].

50. Page Facebook des éditions Inculte, 15 octobre 2017. En ligne, URL: https:// www.facebook.com/editions.inculte/?

ref =search\&_-tn $\mathrm{v}$ [consulté le 15 octobre 2017].

51. David Vrydaghs, « Des cafés aux dancings », COnTEXTES, ${ }^{\circ} 6$, septembre 2009. En ligne, URL : http://journals.openedition.org/contextes/4412 [consulté le 30 octobre 2019].

52. Michel Lacroix, « Un sujet profondément imprégné d'alcool », Art. cit.

53. Anthony Glinoer, « L'orgie bohème », Art. cit.

54. Geneviève Boucher, Pascal Brissette, « De l'alcool à l'écriture et vice versa ", COnTEXTES, $n^{\circ} 6$, septembre 2009. En ligne, URL : http://journals.openedition.org/contextes/4455 [consulté le 30 octobre 2019].

55. Alain Viala, "Posture ", in Anthony Glinoer et Denis Saint-Amand (dir.), Le lexique socius. En ligne, URL : http://ressources-socius.info/index.php/lexique/21-lexique/69-posture [consulté le 02 juin 2020].

\section{RÉSUMÉS}

Depuis les travaux de Jérôme Meizoz, la notion de "posture » connaît un fort succès critique. Mais, si la recherche a analysé les images construites par de nombreux auteurs dans et hors du texte, elle s'est encore peu intéressée à la question des postures collectives. Pourtant, tout groupe littéraire, en tant qu'il occupe une position au sein du champ, est amené à se donner une posture, dès lors qu'on l'envisage comme "une manière singulière d'occuper une position " (Jérôme Meizoz). Il s'agira donc de proposer une première ébauche théorique du concept de "posture collective ", en étudiant ses lieux et ses modes de création et d'exposition ainsi que les jeux de concurrence entre posture individuelle et collective, pouvant provoquer des cas de discordance posturale ou au contraire d'appropriation voire de confiscation de la posture commune. 
Pour mener cette étude, nous aimerions nous appuyer sur l'exemple du collectif Inculte. S'il emprunte à la famille posturale des avant-gardes certains traits de son image collective (camaraderie, esprit potache), il s'en distingue en évitant soigneusement les lieux de définition traditionnelle d'une posture unifiée, en particulier le manifeste et la figure du chef de file. Le défi serait alors d'identifier les modes d'élaboration de cette posture fuyante au sein d'un groupe qui s'attache à promouvoir un geste collectif de création sans homogénéisation dogmatique (esthétique ou politique). À partir de leurs ouvrages collectifs et de la revue Inculte, en explorant les convergences de leurs déclarations, la circulation et la reprise de formules touchant au groupe et à la littérature, l'élection commune d'auteurs-mana formant une bibliothèque Inculte, mais aussi leur présence et leurs interactions sur les réseaux sociaux, il deviendrait ainsi possible de préciser les contours d'un autoportrait diffracté.

Since it has been theorized by Jérôme Meizoz, the notion of "posture" has been a popular concept among critics. But, if researchers have analyzed the images constructed by numerous authors in and out of the text, they have not yet so far been very interested in the question of collective postures. However, any literary group, insofar as it occupies a position within the field, is led to give itself a posture, as soon as one considers it as "a singular way of occupying a position" (Jérôme Meizoz). It will thus be a question of proposing a first theoretical draft of the concept of "collective posture", by studying where and how it may be created and showed as well as how individual and collective posture can compete, causing cases of postural discordance or on the contrary of appropriation even of confiscation of the common posture.

To carry out this study, we would like to rely on the example of the Inculte collective. If it borrows from the postural family of the avant-gardes certain features of its collective image (camaraderie, a sense of humour), it distinguishes itself from it by carefully avoiding the traditional places of definition of a unified posture, in particular the manifesto and the figure of the leader. The challenge would then be to identify the modes of elaboration of this elusive posture within a group that strives to promote a collective gesture of creation without dogmatic homogenization (aesthetic or political). From their collective works and the Inculte magazine, by exploring the convergences of their declarations, the circulation and the resumption of formulas touching the group and the literature, the common election of authors-mana forming an Inculte library, but also their presence and their interactions on the social networks, it would thus become possible to specify the contours of a diffracted self-portrait.

\section{INDEX}

Mots-clés : littérature contemporaine, posture collective, collectif Inculte, groupes littéraires Keywords : contemporary literature, collective posture, Inculte collective, literary groups

\section{AUTEUR}

\section{JEAN-MARC BAUD}

Jean-Marc Baud est agrégé en lettres modernes et docteur en littérature française. Sa thèse, soutenue en 2020, s'intitule « Esthétiques et posture du collectif Inculte (2004-2015) : faire groupe après le siècle des avant-gardes ». Il a également publié différents articles sur les auteurs du collectif, comme Mathieu Larnaudie, Maylis de Kerangal ou Mathias Énard, et a co-organisé en 2019 le premier colloque universitaire sur le football et la littérature, à l'Institut Lumière de Lyon. 